\title{
Prefrontal repetitive transcranial magnetic stimulation as add on treatment in depression
}

\author{
M García-Toro, A Pascual-Leone, M Romera, A González, J Micó, O Ibarra, H Arnillas, \\ I Capllonch, A Mayol, J M Tormos
}

\begin{abstract}
A growing number of studies report antidepressant effects of repetitive transcranial magnetic stimulation (rTMS) in patients with major depression. The hypothesis that high frequency $(20 \mathrm{~Hz})$ rTMS (HF-rTMS) may speed up and strengthen the therapeutic response to sertraline in MD was tested. Twenty eight patients who had not yet received medication for the present depressive episode $(n=12)$ or had failed a single trial of an antidepressant medication $(n=16)$ were started on sertraline and randomised to receive either real of sham HF-rTMS. HF-rTMS was applied to the left dorsolateral prefrontal area in daily sessions (30 trains of $2 \mathrm{~s}, 20-40 \mathrm{~s}$ intertrain interval, at $90 \%$ motor threshold) on 10 consecutive working days. The results suggest that in this patient population, HF-rTMS does not add efficacy over the use of standard antidepressant medication.

(F Neurol Neurosurg Psychiatry 2001;71:546-548)
\end{abstract}

Keywords: major depression; transcranial magnetic stimulation; sertraline; prefrontal area

Repetitive transcranial magnetic stimulation (rTMS) is a non-invasive method of inducing a fairly localised current in the cortex of the human brain. A time varying magnetic field is generated by a brief current pulse passed through a stimulation coil held over the subject's scalp. The magnetic field penetrates the scalp and skull unattenuated. The rapid change of the magnetic field induces a current strong enough to depolarise neurons and thus modify the activity in the targeted cortical area and trans-synaptically in functionally connected brain structures. ${ }^{12}$ If appropriate guidelines are followed rTMS is safe and has minimal side effects. ${ }^{3}$

Most of a growing number of studies on rTMS in depression report antidepressant effects applying high frequency stimulation (HF-rTMS; >1 Hz) over the left prefrontal cortex, whereas others have employed low frequency rTMS (LF-rTMS; <1 Hz) over the right prefrontal cortex (for reviews see George et $a l,{ }^{1}$ Reid et $a l,{ }^{4}$ and Menkes et $\left.a l^{5}\right)$. Nevertheless, few controlled studies have focused on the effect of rTMS as add on treatment to medications in depression. We report a double blind controlled pilot study of left prefrontal HF-rTMS as adjuvant treatment to sertraline in patients with major depression.

\section{Methods}

The local research and ethics review board approved the study. All patients gave their written informed consent before entering the study and after the procedure and objectives had been fully explained to them. We recruited 28 patients older than 18 years of age who had not tried sertraline for the present depression episode and met DSM IV criteria for major depression. $^{6}$ Prospective patients were screened for contraindications for rTMS $^{3}$ including personal or family history of seizures, past neurosurgical procedures, implanted pacemaker, inner ear prosthesis, medication pumps, pregnancy, and unstable medical conditions. Patients with a high suicidal risk, based on a structured diagnostic interview were also excluded.

The patients made a first selection evaluation visit in which, after confirming the DSM-IV diagnosis, a wash out period of 1 week off all medications began. Then, in addition to undergoing rTMS, all these patients were started on sertraline $(50 \mathrm{mg}$ for 2 weeks, later increased, if necessary, depending on clinical response). All 28 patients, except two, were taking benzodiazepines at the time of entry into the study. These were kept unchanged. Two subjects were left handed, and both of them were randomised to receive sham rTMS.

Stimulation was applied to the left dorsolateral prefrontal cortex ${ }^{7}$ using a Dantec Magpro stimulator (Dantec Medical, Medtronic Inc, Minneapolis, MN, USA) and an 8 shaped coil (each wing measuring $8.5 \mathrm{~cm}$ in diameter). Stimulation intensity was $90 \%$ of the motor threshold intensity defined according to the current recommendations of the International Federation of Clinical Neurophysiology. Stimulation frequency was $20 \mathrm{~Hz}$. The coil was centred $5 \mathrm{~cm}$ anterior to and in the same parasagittal plane as the optimal scalp position for activation of the contralateral abductor pollicis brevis muscle. ${ }^{7}$ The stimulation indices used were within current safety guidelines. ${ }^{3}$ 
Table 1 Summary of results

\begin{tabular}{lll}
\hline & Real HF-rTMS & Sham rTMS \\
\hline Patients (n) & 11 & 11 \\
age (y) (mean (SD)) & $43.2(13.1)$ & $45.0(18.3)$ \\
Males & 5 & 5 \\
Inpatients & 3 & 1 \\
Patients with one previous pharmacological & 5 & 6 \\
$\quad$ trial & 7 & \\
Patients with previous episodes & $6.6(4.2)$ & $8.1(12.6)$ \\
Duration of present episode (months) & $25.9(6.4)$ & $26.6(6.4)$ \\
HDRS entry score & $20.1(5.2)(-20.7 \%)$ & $22.3(7.0)(-15.3 \%)$ \\
HDRS 1 week score & $16.1(7.7)(-38.2 \%)$ & $17.9(8.7)(-34.3 \%)$ \\
HDRS 2 week score & $14.3(7.1)(-45.2 \%)$ & $14.5(10.9)(-45.2 \%)$ \\
HDRS 4 week score & $5.0(0.9)$ & $4.3(1.1)$ \\
GCI entry score & $4.2(1.1)(-16.0 \%)$ & $3.9(1.3)(-9.3 \%)$ \\
GCI 1 week score & $3.3(1.4)(-34.0 \%)$ & $3.4(1.6)(-20.9 \%)$ \\
GCI 2 week score & $2.5(1.7)(-50.0 \%)$ & $2.6(1.7)(-39.5 \%)$ \\
GCI 4 week score & $27.09(8.5)$ & $23.18(6.9)$ \\
BDI entry score & $23.1(5.1)(-14.4 \%)$ & $22.3(8.3)(-3.7 \%)$ \\
BDI 1 week score & $19.4(6.7)(-28.1 \%)$ & $21.2(7.9)(-8.2 \%)$ \\
BDI 2 week score & $19.5(7.4)(-27.7 \%)$ & $18.5(11.1)(-20.1 \%)$ \\
BDI 4 week score & & \\
\hline
\end{tabular}

HDRS=Hamilton depression rating scale; GCI=global clinical inventory; BDI=Beck depression inventory. first 2 weeks. At the second week, four of the patients who received HF-rTMS had a $>50 \%$ decrease in HDRS scores, and four had a $>25 \%$ decrease (in the sham HF-rTMS three had a $>50 \%$ decrease in HDRS scores, and five had a $>25 \%$ decrease).

The only side effect of rTMS encountered was slight, self limited, and transitory muscle tension headaches affecting three of the 11 patients that received real HF-rTMS.

\section{Discussion}

In combination with antidepressant medication (sertraline), real rTMS resulted in a similar antidepressant effect to sham rTMS. In these patients real rTMS seems to have added nothing to the medication regimen alone. It is possible that HF-rTMS was not effective. Alternatively, patients with major depression may have benefited from sertraline enough to obscure a small additional benefit from the rTMS. The placebo effect of rTMS may also have contributed to abolish statistical significance. The lack of a control group receiving medication and no rTMS precludes us from making this distinction. Nevertheless, our results suggest a slight non-statistical tendency for the therapeutic effect for the combination of real rTMS with medication to be faster than that of sham rTMS and medication (table 1). The main limitation of this trial is the relatively small sample size.

There are several studies available which have used rTMS in patients with major depression while they were taking antidepressant drugs. In all these trials the antidepressant effects and potential side effects of rTMS seemed unaffected by the concurrent intake of medication. Conca et al were the first to specifically study the potential benefit of rTMS as add on therapy to antidepressant medication in patients with major depression. ${ }^{11}$ They conducted a controlled clinical trial on 24 patients randomised to receive antidepressant medication with or without concurrent rTMS add on. Patients receiving rTMS add on showed a faster remission of depressive symptoms. Differences between the two patient groups became statistically significant already after the third rTMS session and grew by the end of the study. Our results do not replicate the findings of Conca et al, perhaps because in their study the lack of sham rTMS control resulted in a rather different protocol for both patient groups and made the study essentially an open design. ${ }^{11}$ Other studies using rTMS as an augmentation technique to antidepressant medication have found positive and negative results, but are also difficult to compare with ours because of important differences in the methodology and sample used (medication resistant patients). ${ }^{12} 13$

In conclusion, in this sample of non-resistant patients with major depression, HF-rTMS seems to have no adjunctive benefit associated with sertraline. AP-L is supported in part by the National Alliance for Research
in Schizophrenia and Depression (NARSAD), the NAMI in Schizophrenia and Depression (NARSAD), the NAMI
Stanley Vada Foundation, and the National Institute of Mental Stanley 
1 George MS, Lisanby SH, Sackeim HA. Transcranial magnetic stimulation Applications in neuropsychiatry. magnetic stimulation. Applications

2 Pascual-Leone A, Bartres-Faz D, Keenan JP. Transcranial magnetic stimulation: studying brain-behavior relationship by induction of "virtual lesions". Transactions of the Royal Philosophical Society 1999;354:1229-38

3 Wassermann EM. Risk and safety of repetitive transcranial magnetic stimulation: report and suggested guidelines from the International Workshop in the Safety of Repetitive Transcranial Magnetic Stimulation, June 5-7, 1996. Electroencephalogr Clin Neurophysiol 1998;108:1-16.

4 Reid PD, Shajahan PM, Glabus MF, et al. Transcranial magnetic stimulation in depression. $\mathrm{Br} f$ Psychiatry 1998;24:449-52

5 Menkes DL, Bodnar P, Ballesteros RA, et al. Right frontal lobe slow frequency repetitive transcranial magnetic stimulation (SF r-TMS) is an affective treatment for depression: a case-control pilot study of safety and efficacy. $\mathcal{F}$ Neurol Neurosurg Psychiatry 1999;67:113-15.

6 American Psychiatric Association. Diagnosis and statistical manual of mental disorders. 4th ed. Washington, DC: APA, 1994.
7 Pascual-Leone A, Rubio B, Pallardó F, et al. Rapid rate transcranial magnetic stimulation of left dorsolateral prefrontal cortex in drug-resistant depression. Lancet 1996;348:2337.

8 Hamilton M. A rating scale for depression. 7 Neurol Neurosurg Psychiatry 1960;23:56-62.

9 Guy W. Early clinical drug evaluation (ECDEU) assessment manual. Rockville: National Institute Mental Health, 1976.

10 Beck AT, Steer RA. Manual for the revised Beck depression inventory. San Antonio: Psychological Corporation, 1987.

11 Conca A, Koppi S, Koning P, et al. Transcranial magnetic stimulation: a novel antidepressive strategy? Neuropsychobiology 1996;34:204-7

12 Figiel GS, Epstein C, McDonald WM, et al. The use of rapid-rate transcranial magnetic stimulation (rTMS) in refractory depressed patients. $\mathcal{F}$ Neuropsychiatry Clin Neurosci $1998 ; 10: 20-5$.

13 Padberg F, Zwanzger P, Thoma H, et al. Repetitive transcranial magnetic stimulation (rTMS) in pharmacotherapyrefractory major depression: comparative study of fast, slow and sham rTMS. Psychiatry Res 1999;88:163-71.

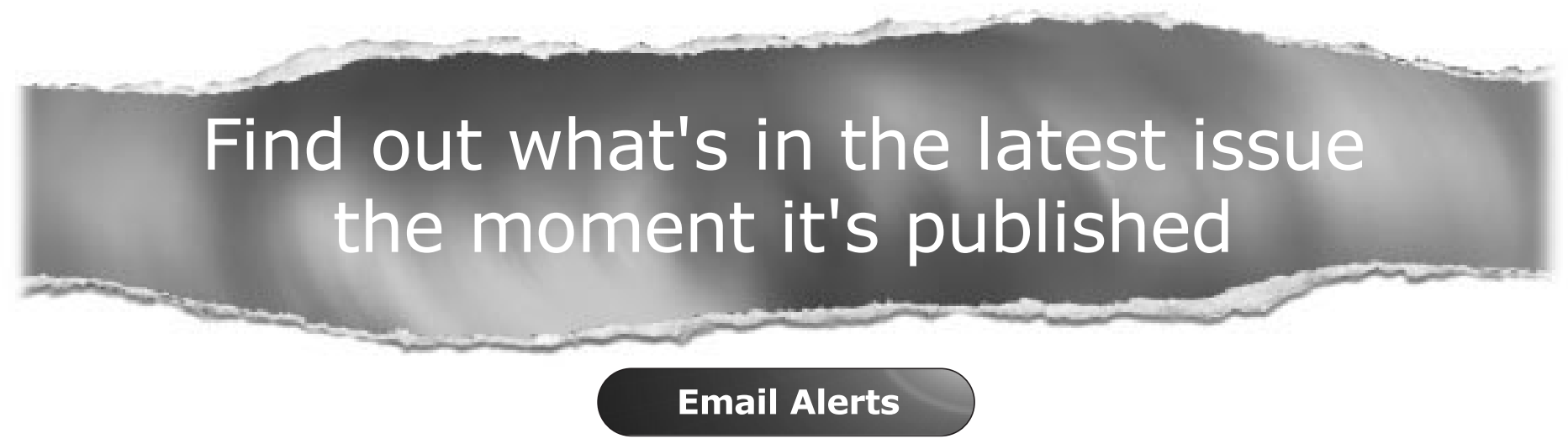

Sign up to receive the table of contents by email every month. You can select from three alerts: Table of Contents (full), TOC Awareness (notice only); Journal of Neurology, Neurosurgery, and Psychiatry related announcements.

www.jnnp.com 